\title{
Mental Health Education's \\ Effect on Physiotherapy Students' Emotional Attitudes towards Working with Patients with Mental Health Disorders
}

\section{Bev Sarin ${ }^{1}$, Richard Walters ${ }^{2}$, Tony Everett ${ }^{1}$}

'Department of Physiotherapy, School of Healthcare Studies, Cardiff University

2Department of Physiotherapy, Torbay Hospital, South Devon Healthcare NHS Foundation Trust, UK

Contact: sarinbj@cardiff.ac.uk

\section{Introduction}

The development of an effective therapeutic relationship is based to a large extent on the degree to which a Healthcare worker can empathise and relate to the individual in their care (Shattell et al, 2007).

Empathy is an emotional response to another person (Norfolk et al, 2007) and can be adversely affected by negative emotional biases. These biases may have developed because of a lack of understanding or prejudice about Mental Health Disorders (MHD) or fear of their behavioural consequences, and so go on to act as barriers to the development of appropriate empathic communication and hindering the ability to develop therapeutic rapport.

Physiotherapy students may enter undergraduate training with belief and emotional biases, and fears in relation to MHD. To prepare and enable students to engage effectively with their patients, Physiotherapy Education needs to address and challenge the cognitive and affective aspects of students' understanding as part of their professional development, this in turn will support their engagement with people with MHD.

The inclusion of a specific Mental Health Teaching Block (MHTB) within the undergraduate physiotherapy training in the Cardiff School of Physiotherapy has been included in the curriculum to address this need.

\section{Aims}

To explore the emotional attitudes that level 2 physiotherapy students have in association with their engagement with patients with Mental Health Disorders.

To identify changes that occur in these emotional attitudes following a MHTB.

\section{Methodology}

75 Level 2 (preclinical) students from the Physiotherapy BSc (Hons) programme at the Cardiff School of Physiotherapy (cohort size of 75 ) volunteered to participate in the study.

A questionnaire was used to deliver patient scenarios in the form of vignettes. A case study of a man who has suffered a fractured ankle, was taken from an outpatient department setting. This, the 'no MHD' scenario, was used to provide a baseline of emotional reactions. To this scenario, details about 3 Mental Health Disorders are added to provide co-morbid scenarios. The conditions added were Schizophrenia (Patient scenario below), Depression and Dementia.

'John has been referred to you for outpatient physiotherapy following an ORIF (open reduction internal fixation) for an ankle fracture. You have read the medical notes from the ward, which amongst other information includes that a few years ago after a number of paranoid psychotic episodes he was diagnosed with schizophrenia.

Fortunately he has not had any psychotic symptoms since diagnosis.

Please form a picture of how you would expect John to be and rate how likely you are to feel the following emotions when treating him

Subjects were asked to grade 11 emotions ( 3 positive, 8 negative) in association with each of the 4 scenarios, using a 7 point Likert scale $(1=$ very unlikely to $7=$ very likely $)$ Questionnaires were completed 2 days before and 1 week after the MHTB. Ethical approval was gained through UWCM.

Analysis of the results identified trends in the data using SPSS statistical package for mean, median and range of scores. Differences between the pre and post MHTB responses were analysed using the Wilcoxon Signed-Ranks Test.

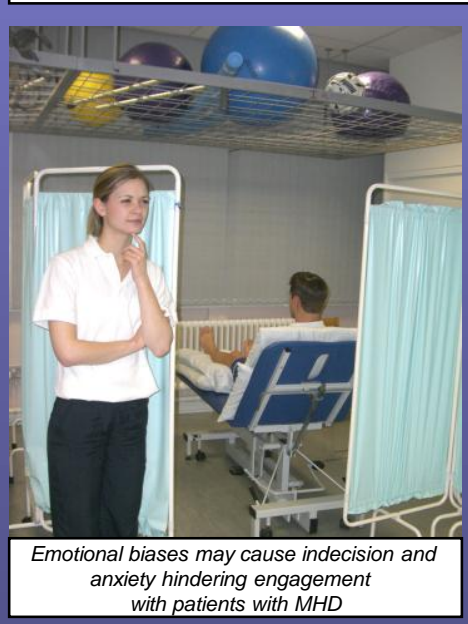

\section{Results}

There was a return rate of $75 \%$, with 56 paired questionnaires providing a comparison between pre and post MHTB responses

Comparison of the emotional attitudes that students associated with the patient scenarios between pre and post MHTB questionnaires provided the following results:

The highest negative emotions were associated with the schizophrenia scenario, which also showed the most significant overall reduction after the MHTB.

Of the positive emotions, there was an increase in expectation of feeling relaxed with the schizophrenia scenario $(p=0.015)$, but responses indicated that they were less likely to feel empathy for 'no MHD' $(p=0.029)$, depression $(p=0.004)$, dementia $(p=$ $0.013)$ or schizophrenia $(p=0.006)$

Of the negative emotions, there was a reduced likelihood of feeling fear \& wariness with schizophrenia $(p=0.012$ and $p=0.000$ respectively). There was a reduction in feelings of nervousness with the depression $(p=0.013)$, dementia $(p=0.003)$ and schizophrenia scenarios $(p=0.014)$, and anxiousness with dementia $(p=0.003)$ and schizophrenia scenarios $(p=0.017)$ There was also a reduction in understanding for the 'no MHD' scenario $(p=0.016)$ post MHTB.

Results for feelings of anger and disgust were all within the 'unlikely' range for all scenarios, both pre and post MHTB. Feelings of sadness \& nothing showed no significant change.

\section{Conclusion}

It was interesting to note that students felt less understanding for John in the 'no MHD' scenario following the MHTB. It can only be speculation to whether this is a result of the evaluation of the 'no MHD' scenario against the more complex MHD scenarios or, as the students are preclinical, the re evaluation of patient interaction as a whole in light of the new skills they have learnt.

Increasing knowledge and understanding of MHD as a result of the MHTB has lead to an overall reduction of negative and increase in positive emotions associated with engagement with a patient with a concomitant MHD. Although there was a reduction of empathy recorded for all scenarios post MHTB, this may be due to a misinterpretation of empathy as 'sympathy'. Despite this apparent reduction of empathy, the foundation of therapeutic relationships (Norfolk et al, 2007), the positive shift in emotional biases made by the students would indicate a basis on which to develop sound therapeutic relationships. This shift in emotional associations may allow for greater objectivity in decision-making, widening of opportunities for enhancing communication, and enable a greater understanding of the 'patient experience' by the student when working with patients with MHD, in which ever healthcare environment they are encountered.

\section{References}

Norfolk T, Birdi K, Walsh D (2007) The role of empathy in establishing rapport in the consultation: a new model Medical Education 41: 690-697

Shattell MM, Starr SS, Thomas SP (2007) 'Take my hand, help me out': Mental health service recipients' experience of the therapeutic relationship International Journal of Mental Health Nursing 16: 274-284 